E3S Web of Conferences 2, 02005 (2014)

DOI: $10.1051 /$ e3sconf/ 20140202005

(C) Owned by the authors, published by EDP Sciences, 2014

\title{
I cambiamenti climatici: la sfida del XXI secolo
}

\section{Climate changes: the XXI Century challenge}

\author{
Stefano Caserini ${ }^{\text {a }}$ \\ Politecnico di Milano, D.I.C.A. Sez. Ambientale, Piazza Leonardo da Vinci 32, 20133 Milano, Italy
}

\begin{abstract}
Riassunto. È ampio il divario fra le azioni necessarie per evitare un surriscaldamento dannoso del pianeta e quelle decise per contrastarlo. Più passa il tempo più le visioni pessimiste acquistano motivazioni e argomenti validi. Per trasformare radicalmente in pochi decenni un sistema energetico e un rapporto predatorio verso le risorse del pianeta è necessario riconoscere la crisi climatica nella sua dimensione sistemica, nel suo essere prima di tutto un problema di giustizia, di equità anche verso le generazioni future. La scienza del clima dovrebbe aiutare a capire la portata della sfida chiarendo meglio le conseguenze delle scelte odierne per le generazioni future. Ma uno sforzo molto più grande è quello di recuperare le ragioni per occuparci, come individui e comunità, dei nostri posteri. Capire la posta in gioco sul tema del cambiamento climatico richiede non solo più conoscenza, ma di accettare l'esistenza dei limiti planetari, di ridefinire le aspettative amane, nonché un livello di intelligenza emotiva oggi insufficiente.
\end{abstract}

\begin{abstract}
There is a large gap between the actions required to avoid a dangerous warming of the planet, and those already decided to contrast it. The more time passes, the more the pessimistic visions obtain valid motivations and reasons. In order to radically change in few decades an energetic system and a predatory attitude towards the planet's resources, it is necessary to recognize the climate crisis as a systemic one, as a problem of justice and equity for the future generations. The climate science should help to understand the importance of this challenge, by emphasizing the consequences of today's decisions for the future generations. To recover our reasons to deal with posterity, both as individuals and community, is a bigger endeavor. To understand the importance of the climate change requires not only more knowledge, but also the acceptance of the planetary limits, the redefinition of human expectations, and also a level of emotional understanding that is not sufficient today.
\end{abstract}

\footnotetext{
${ }^{a}$ Corresponding author: Stefano.caserini@polimi.it
} 


\section{Introduzione}

Alla fine di un'intervista o di una conferenza pubblica sul tema dei cambiamenti climatici arriva quasi sempre la domanda: ma quali possono essere oggi i motivi di speranza?

Una risposta onesta, che non si rifugi nella facile retorica delle magnifiche sorte e progressive, ma sappia reggere lo sguardo con la realtà del surriscaldamento globale, è difficile. Mentre le evidenze scientifiche si stanno accumulando e ormai formano un quadro coerente, seppur con diversi punti ancora incerti e da meglio precisare, le azioni già messe in campo o che si delineano nei prossimi anni sono largamente insufficienti a contrastare l'aumento delle emissioni di gas climalteranti; le possibilità di una rapida correzione di rotta, che assicuri il rispetto degli obiettivi di incremento delle temperature che sono stati alla base di quindici anni di negoziazioni internazionali, sia fanno via via più ridotte.

\section{II consenso scientifico}

Numerosi sono i rapporti scientifici che hanno confermato il consenso della comunità scientifica sul tema del surriscaldamento globale. Rimanendo ai documenti più recenti, è possibile citare il "Quinto Rapporto di Valutazione" dell'Intergovernmental Panel on Climate Change [1], l'organismo ONU a cui collaborano migliaia di scienziati che periodicamente effettua una sintesi della letteratura scientifica disponibile, o lo statement "Human-induced climate change requires urgent action" dell'American Geophysical Union [2], una delle principali organizzazione scientifiche mondiali.

Pur se in passato numerose sono state le voci che hanno negato un fondamento alle preoccupazioni per i pericoli dei cambiamenti climatici (una rassegna è disponibile in [3]), se si valuta la letteratura scientifica degli ultimi 10 anni emerge come il dibattito sulle responsabilità umane in merito ai recenti e futuri cambiamenti climatici è ormai anacronistico. Si tratta di una produzione scientifica vastissima, che riguarda non solo la descrizione fenomenologica del problema, ma anche le necessarie strategie di adattamento e di mitigazione, in grado di fornire risposte ai dubbi molto popolari nei mass media sulla realtà del riscaldamento in atto, sulla determinante influenza umana e sulla pericolosità dei danni attesi nei prossimi decenni. Al di là delle variazioni di breve periodo delle temperature globali della superficie dell'atmosfera, che possono trarre in inganno, è l'imponente mole di dati sperimentali e di simulazioni modellistiche a motivare il grande consenso scientifico sul fatto che le emissioni di gas serra stanno ancora aumentando, il pianeta si sta riscaldando, i ghiacci si stanno riducendo in estensione e volume, il livello dei mari sta crescendo. Il cuore del dibattito è ormai altrove, ad esempio quanto potrà in futuro riscaldarsi il pianeta, in relazione alle emissioni umane di gas serra; quali potrebbero essere $\mathrm{i}$ feedback positivi su lunghe scale temporali in grado di amplificare questo riscaldamento [4]; nonché l'esistenza di possibili "tipping points", soglie critiche che se superate potrebbero variare bruscamente alcuni parametri climatici o impedire il ritorno alle condizioni precedenti [5].

\section{La realtà del riscaldamento globale}

Il riscaldamento globale sta già interferendo con i sistemi ecologici e le attività umane. I danni e le sofferenze che i cambiamenti climatici stanno già causando sono dovuti al clima che si fa sempre più estremo [6], con ondate di calore prolungate, precipitazioni più irregolari e intense, con scompensi per le risorse idriche e riduzioni delle rese agricole [7]. Solo le ricerche più importanti riescono a sfondare il silenzio grandi mezzi di comunicazione sul tema dei cambiamenti climatici, di cui si sente parlare sono in occasioni di qualche alluvione o ondata di calore eccezionale. Ma è scarso l'interesse per la reale dimensione del problema climatico, in un'ottica plurisecolare, come inizio di processi (la fusione delle calotte polari, l'innalzamento del mare) pericolosi in quanto inarrestabili una volta avviati. 
Anche grafici eloquenti come quello mostrato nella Figura 1, che rivela con chiarezza e brutalità quanto l'aumento delle temperature previsto per questo secolo potrà essere elevato se confrontato con le variazioni delle temperature degli ultimi 1500 anni, sono sconosciuti non solo all'opinione pubblica e ai decisori politici, ma molti studenti che si laureano in materie scientifiche.

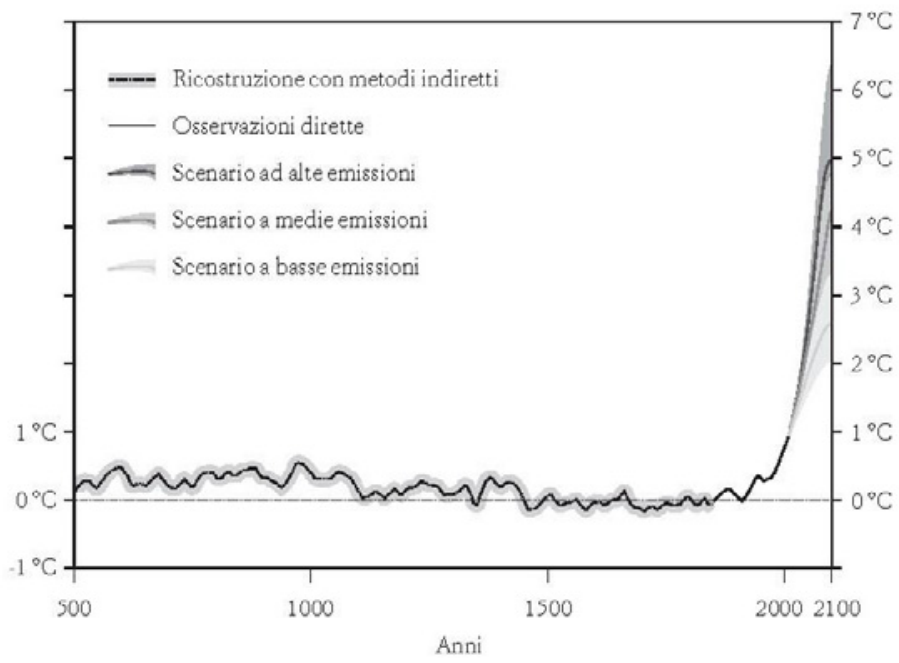

Figura 1. Variazione delle temperature medie globali (rispetto alla media 1800-1900) ricostruite, osservate e proiezioni per il futuro. (Fonte: www.copenhagendiagnosis.org).

Il recentissimo Quinto Rapporto dell'IPCC ha confermato l'accelerazione della perdita di massa nella calotta glaciale della Groenlandia (da 34 Gt/anno nel 1992-2001 a 215 Gt/anno nel 2002-2011), l'incremento del tasso di innalzamento del livello globale medio del mare $(1.7 \mathrm{~mm} /$ anno nel periodo 1901-2010 e $3.2 \mathrm{~mm} /$ anno nel 1993-2010), ha ribadito che molti dei cambiamenti che si stanno verificando (alla temperatura dell'aria, degli oceani, nel ciclo dell'acqua, nel livello dei mari, nella criosfera, in alcuni eventi estremi e nell'acidificazione oceanica) persisteranno per molti secoli.

Una parte del riscaldamento del pianeta è già deciso dagli attuali livelli dei gas serra, ma non si è ancora verificato per l'inerzia del sistema: indipendentemente dalle azioni che saranno messe in campo nei prossimi decenni, le temperature medie globali del pianeta saliranno ancora, si avvicineranno alle soglie critiche.

L'agire combinato delle variazioni climatiche e della perdita di biodiversità legata all'antropizzazione del territorio metterà sotto stress molti sistemi ecologici e ha spinto ecologi e biologi a parlare del rischio di una "sesta grande estinzione".

I tempi lenti delle variazioni del clima futuro sono tempi rapidi per un paleoclimatologo o un geologo, abituati a ragionare di ere e periodi geologici, scale temporali di millenni e milioni di anni. Sono tempi lentissimi per un meteorologo, o per un politico, abituati ad interessarsi alle variazioni che avvengono in giorni o settimane, una scala temporale molto più vicina ai tempi televisivi che alle dinamiche climatiche.

\section{La variabilità che confonde}

I mass media sembrano incapaci di capire anche i concetti più semplici e basilari del problema del cambiamento climatico, come quello di tendenza climatica e di variabilità. Ancora nell'estate del 2013, un lieve recupero dell'estensione del ghiaccio marino artico ha portato a titoli di quotidiani in cui si descrivono fantomatiche "inversioni di tendenza" o recuperi inattesi, mentre la tendenza di diminuzione è chiara (l'estensione del ghiaccio marino artico in settembre, quando si raggiunge il minimo, si è quasi dimezzata in poco più di trent'anni). 
Il temporaneo rallentamento nell'aumento delle temperature globali alla superficie negli ultimi anni, seppur ampiamente spiegato all'interno della normale variabilità del sistema climatico e per un maggiore riscaldamento degli oceani [8], ha dato l'appiglio per illusioni di un ridimensionamento del problema. In realtà, l'aumento delle temperature globali negli ultimi quattro decenni è stato molto rilevante, da $0,13^{\circ} \mathrm{C} \mathrm{a} 0.22{ }^{\circ} \mathrm{C}$ per decennio, seppur con una grande variabilità interannuale. Il primo decennio del XXI secolo è il più caldo da quando esistono dati di rilevazione delle temperature.

La storia dell'uomo è da sempre una storia di modifica del territorio e dell'ambiente, ma questa modifica non ha paragoni col passato per la sua rapidità, la sua entità e la sua vastità. Una recente ricostruzione delle temperature dell'Olocene (circa gli ultimi 11.000 anni) pubblicata su Science [9], ha mostrato come il recente riscaldamento globale sia senza precedenti negli ultimi 1500 anni e le temperature attuali siano superiori a quelle registrate nel 75\% dell'Olocene (Figura 2).

C'è una gran voglia di non credere a quanto sta succedendo, di accusare gli scienziati di essere allarmisti; ma non è chiara la differenza fra l'allarmismo e la legittima preoccupazione per lo stato del pianeta.

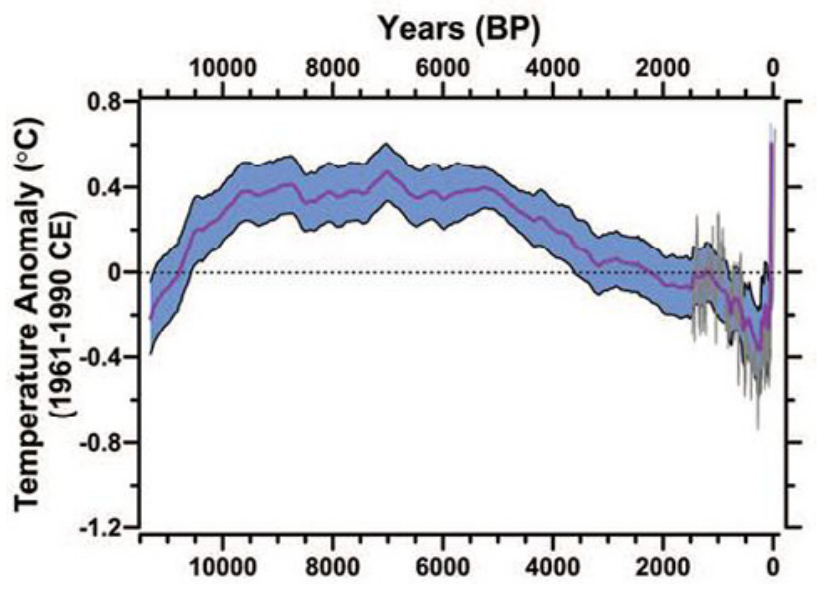

Figura 2. Ricostruzione delle temperature globali negli ultimi 11.300 anni. Fonte: [9].

\section{L'aumento dei motivi di preoccupazione}

Mentre i media, spesso a causa di specifiche campagne di disinformazione, continuano a definire allarmistiche le proiezioni sul clima futuro e a prospettare inesistenti "retromarce" della comunità scientifica, diversi lavori hanno mostrato come la comunità scientifica può, al limite, essere accusata del contrario, di essere stata troppo cauta, di aver proposto una visione edulcorata del problema [10]. I motivi sono legati al rigoroso rispetto delle norme scientifiche che prevedono di fondare le affermazioni su prove inattaccabili, ma anche lo scetticismo di fondo, la razionalità, il distacco e la moderazione che caratterizzano il metodo scientifico. Secondo James Hansen, uno dei più grandi climatologi viventi, questo potrebbe portare ad una sorta di reticenza scientifica, in quanto gli scienziati tendono ad evitare di comunicare l'effettiva portata delle implicazioni dei dati e delle proiezioni che essi, meglio di altri, comprendono [11].

Con l'avanzamento della comprensione degli impatti dei cambiamenti climatici i motivi di preoccupazione aumentano, e l'interferenza dannosa delle attività umane con il clima è oggi associata a minori incrementi delle temperature globali [12]. Iniziano a essere discussi nella letteratura scientifica i primi segnali di fenomeni il cui dispiegamento avrebbe conseguenze enormi, come la perdita di massa glaciale della calotta Antartica [13] o la fusione del permafrost artico [14].

Nel frattempo, l'andamento delle emissioni globali non sembra risentire delle timide politiche di mitigazione decise a livello internazionale. La crisi economica degli anni 2008-2009 ha avuto un 
effetto molto limitato sulle emissioni globali dall'uso dei combustibili fossili, che hanno raggiunto nel 2012 il nuovo record, con un aumento dell'1,4\% rispetto al 2011 e del 4,4\% rispetto al 2010 [15]. L'incremento medio della $\mathrm{CO}_{2}$ in atmosfera nel 2012 a Manua Loa è stato di 2,6 ppm, il secondo fra i valori più elevati mai registrati da quando sono iniziate le misure dirette in continuo.

\section{II ritardo della politica}

È ormai evidente il divario fra le politiche di mitigazione, ossia le azioni necessarie per ridurre le emissioni di gas climalteranti, e le politiche stabilite o in corso di decisione. Il grande, enorme ritardo della politica ha tante motivazioni.

Il primo è la ricerca di consenso politico di breve periodo che caratterizza l'agire dei decisori politici, il loro rappresentare gli interessi prevalenti e momentanei della società.

Il secondo è la povertà come ostacolo all'innovazione e alla diffusione di tecnologie pulite. Ancora un miliardo di persone hanno un accesso all'energia molto limitato e la protezione del clima non può per loro essere prioritaria, visti i guadagni di benessere legati all'accesso all'energia. D'altronde, l'attuale sistema economico non è stato pensato per garantire parità di accesso alle risorse e un benessere durevole per tutti, ed è quindi del tutto comprensibile che senza seri correttivi possa portare a danni rilevanti per l'ambiente e gli esseri umani, con gravi squilibri geografici e generazionali. Il libero mercato o il perseguimento degli interessi nazionali dei singoli Stati non sono utili per obiettivi ambiziosi di stabilizzazione delle temperature globali.

Infine, un altro fattore che spiega la grande inerzia del cambiamento, il ritardo nelle azioni di trasformazione dei sistemi produttivi e dei comportamenti individuali, è la mancanza nella maggior parte della popolazione delle informazioni basilari sulla questione clima. E non è solo un problema di informazioni o educazione scientifica: ci sono dimostrati meccanismi psicologici e sociologici che portano a rimuovere, anche inconsciamente, fatti e azioni scomode, che provocano ansia, inquietudine.

La lentezza del cambiamento e i messaggi tra loro dissonanti veicolati dai mass media generano una sensazione di impotenza diffusa, se non paralisi: l'utente-spettatore che ascolta gli scienziati contrapporsi sullo schermo non sa più a chi credere e su quale base agire; percepisce l'aleatorietà e discutibilità di ogni posizione scientifica e non sa reagire, né ha spesso gli strumenti e le competenze per discernere e farsi un'idea propria su temi così complessi, anche perché spesso non arricchisce il suo sapere con contributi culturali non provenienti dalla televisione.

Le percezioni soggettive possono solo parzialmente essere informative su un problema, quello del clima, caratterizzato da una grande inerzia temporale. La paura non sembra un fattore che porta ad un maggior coinvolgimento nelle azioni di mitigazione [16], e gli shock causati da eventi estremi non sembrano determinare necessariamente più consapevolezza [17]. L'irregolarità del fenomeno e i fattori di disturbo rendono importante un'interpretazione non solo dei singoli eventi, magari basata su sensazioni individuali, temporanee, ma su una lettura globale dei processi in corso.

Negli ultimi anni, si è giunti a teorizzare, quale extrema ratio, una pedagogia delle catastrofi: paradossalmente, gli uomini potrebbero cambiare se e solo se colpiti direttamente da eventi altamente stressanti (ma non letali, o almeno non per tutti...), tali da costringerli a generare e ricercare nuovi apprendimenti, vere e proprie conversioni dei loro stili di vita e di pensiero sul pianeta. Una potente ristrutturazione cognitiva, insomma [18].

\section{La dimensione della sfida}

È quindi evidente che il cambiamento climatico è oggi una grande questione morale, etica, un problema di giustizia, di equità [19]: coloro che sono e saranno più duramente colpiti, le generazioni future, le persone più povere del pianeta, le specie non umane, sono i meno responsabili. La rimozione, o per lo meno la forte sottovalutazione su scala globale del problema del cambiamento climatico è dovuta al fatto che la "verità scomoda" del cambiamento climatico mostra da un particolare punto di vista l'ingiustizia su cui si regge l'attuale sistema economico, che permette 
l'accaparramento indebito di risorse scarse da parte di un numero relativamente piccolo di individui. E una risorsa scarsa è la capacità dell'atmosfera di assorbire i gas climalteranti. La crisi climatica ripropone la domanda sul senso dell'inseguimento continuo della crescita delle produzioni e dei consumi della nostra società; coinvolge un livello profondo della nostra vita, in quanto si tratta di ridefinire i limiti delle aspettative umane.

Accettare la realtà del riscaldamento globale causato dalle attività umane richiede la capacità di autocritica e di dubitare di sé stessi e dei propri miti. Richiede la disponibilità ad accettare la responsabilità personale per gli errori collettivi e ad accettare il bisogno di grandi cambiamenti culturali e sociali [17]. Per un cambiamento di questo tipo, che ha anche forti opposizioni negli interessi costituiti, le motivazioni fornite dalla scienza non sono sufficienti. Come affermato anche nell'editoriale dell'ottobre 2013 della rivista Nature Climate Change, più che maggiori dati e informazioni serve un'azione concertata nazionale ed internazionale, che coinvolga attivamente anche gli scienziati.

La scienza del clima dovrebbe aiutare a capire la portata della sfida chiarendo meglio le conseguenze per le generazioni future delle scelte odierne. Ma uno sforzo molto più grande è quello di recuperare le ragioni per occuparci, come individui e comunità, dei nostri posteri. Capire la posta in gioco sul tema del cambiamento climatico richiede non solo più conoscenza, ma un livello di intelligenza emotiva oggi insufficiente.

La sfida dei cambiamenti climatici non è quindi solo una questione scientifica e tecnologica, richiede una narrazione che permetta di far uscire la questione climatica dalle stanze dei centri di ricerca per farne una questione su cui definire una nuova identità planetaria.

\section{References}

1. IPCC (2013) Fifth Assessment Report (AR5) Climate Change 2013: The Physical Science Basis. Intergovermental Panel on Climate Change, www.ipcc.ch

2. AGU (2013) Human-induced climate change requires urgent action. American Geophysical Union, Washington DC, August 2013.

3. Caserini S. (2009) Guida alle leggende sul clima che cambia. Quando la scienza diventa opinione. Edizioni Ambiente, Milano. Si veda altresì il blog www.climalteranti.it.

4. Lunt D.J. et al, (2010) Earth system sensitivity inferred from Pliocene modelling and data. Nature Geoscience, 3, 60-64.

5. Lenton T.M. et al. (2008) Tipping elements in the Earth's climate system. PNAS-Proceedings of the US National Academy of Sciences, 105, 1786-1793.

6. Coumou D., Rahmstorf S. (2012) A decade of weather extremes. Nature Climate Change, 2, 491496.

7. Lobell D.B., Schlenker W., Costa-Roberts J. (2011) Climate trends and global crop production since 1980. Science, 333, 6042, 616-620.

8. Kosaka Y., Xie S.P. (2013) Recent global-warming hiatus tied to equatorial Pacific surface cooling. Nature.

9. Marcott S.A., Shakun J.D., Clark P.U., Mix A.C. (2013) A reconstruction of regional and global temperature for the past 11,300 years. Science 339, n. 6124, 1198-1201.

10. Brysse K., Oreskes N., O’Reilly J., Oppenheimer M. (2013) Climate change prediction: Erring on the side of least drama? Global Environmental Change 23, 327-337.

11. Hansen J. (2010) Tempeste. Edizioni Ambiente, Milano

12. Smith J.B. et al. (2009) Assessing dangerous climate change through an update of the Intergovernmental Panel on Climate Change (IPCC) "reasons for concern". PNAS 106, 11, 41334137

13. Miles B.W.J., Stokes C.R., Vieli A., Cox N.J. (2013) Rapid, climate-driven changes in outlet glaciers on the Pacific coast of East Antarctica. Nature 500, 563-566. 
14. Cory R.M., Crump B.C., Dobkowski J.A., Kling G.W. (2013) Surface exposure to sunlight stimulates CO2 release from permafrost soil carbon in the Arctic. PNAS 110, 9, 3429-3434.

15. IEA (2013) World Energy Outlook Special Report 2013: redrawing the energy climate map. International Energy Agency, Paris

16. O’Neill S., Nicholson-Cole S. (2009) "Fear Won't Do It". Promoting positive engagement with climate change through visual and iconic representations. Science Communication, 30, 3, 355379.

17. Marshall G. (2013) Reasons why climate disasters might not increase concern about climate change. Climate Change Denial, 6 novembre.

18. Caserini S., Euli E. (2012) Imparare dalle catastrofi. Guida galattica per sopravvivere al futuro. Altraeconomia Edizioni.

19. Markowitz E.M., Shariff A.F. (2012) Climate change and moral judgement. Nature Climate Change, 2, 243-247. 